\title{
Clinical Strains of Pseudomonas aeruginosa Secrete LasB Elastase to Induce Hemorrhagic Diffuse Alveolar Damage in Mice
}

\author{
Yajie Zhu (D) \\ Xiaoli Ge' \\ Di Xie ${ }^{\prime}$ \\ Shangyuan Wang' \\ Feng Chen ${ }^{2}$ \\ Shuming Pan' \\ 'Department of Emergency Medicine, \\ Xinhua Hospital Affiliated to Shanghai \\ Jiao Tong University School of Medicine, \\ Shanghai, 200092, People's Republic of \\ China; ${ }^{2}$ Division of Medical Microbiology, \\ Department of Clinical Laboratory, \\ Xinhua Hospital Affiliated to Shanghai \\ Jiao Tong University School of Medicine, \\ Shanghai, 200092, People's Republic of \\ China
}

Correspondence: Shuming Pan

Department of Emergency Medicine,

Xinhua Hospital Affiliated to Shanghai Jiao

Tong University School of Medicine,

No. 1665, Kongjiang Road, Yangpu

District, Shanghai, 200092, People's

Republic of China

Tel +862I 25076045

Fax +862165030840

Email panshuming@xinhuamed.com.cn

Feng Chen

Division of Medical Microbiology,

Department of Clinical Laboratory,

Xinhua Hospital Affiliated to Shanghai Jiao

Tong University School of Medicine,

No. I665, Kongjiang Road, Yangpu

District, Shanghai, 200092, People's

Republic of China

Tel +862I 25078999

$\mathrm{Fax}+862165030840$

Email chenfeng@xinhuamed.com.cn
Background: Acute lung injury and acute respiratory distress syndrome (ALI/ARDS) are most often caused by bacterial pneumonia and characterized by severe dyspnea and high mortality. Knowledge about the lung injury effects of current clinical bacterial strains is lacking. The aim of this study was to investigate the ability of representative pathogenic bacteria isolated from patients to cause ALI/ARDS in mice and identify the major virulence factor.

Methods: Seven major bacterial species were isolated from clinical sputum and unilaterally instilled into the mouse airway. A histology study was performed to determine the lung injury effect. Virulence genes were examined by PCR. Sequence types of $P$. aeruginosa strains were identified by MLST. LC-MS/MS was used to analysis the bacterial exoproducts proteome. LasB was purified through a DEAE-cellulose column, and its toxicity was tested both in vitro and in vivo.

Results: Staphylococcus aureus, Streptococcus pneumoniae, Streptococcus agalactiae, Acinetobacter baumannii, Klebsiella pneumoniae, Pseudomonas aeruginosa and Escherichia coli were randomly separated and tested 3 times. Among them, gram-negative bacteria have much more potential to cause acute lung injury than gram-positive bacteria. However, P. aeruginosa is the only pathogen that induces diffuse alveolar damage, hemorrhage and hyaline membranes in the lungs of mice. The lung injury effect is associated with the excreted LasB elastase. Purified LasB recapitulated lung injury similar to $P$. aeruginosa infection in vivo. We found that this was due to the powerful degradation effect of LasB on the extracellular matrix of the lung and key proteins in the coagulation cascade without inducing obvious cellular apoptosis. We also report for the first time that LasB could induce DIC-like coagulopathy in vitro.

Conclusion: P. aeruginosa strains are most capable of inducing ALI/ARDS in mice among major clinical pathogenic bacteria tested, and this ability is specifically attributed to their LasB production.

Keywords: unilateral lung injury, LasB elastase, Pseudomonas aeruginosa, ALI/ARDS

\section{Introduction}

Acute lung injury (ALI) and its more serious form acute respiratory distress syndrome (ARDS) are major causes of death in the intensive care unit (ICU). Despite decades of efforts, the pathogenesis of ALI/ARDS remains unclear and an effective treatment has not been developed. ${ }^{1}$ Diffuse alveolar damage (DAD), hyaline membrane formation, alveolar bleeding and bilateral lung inflammation, which are typical pathological features found in ALI/ARDS patients, were poorly recapitulated in animal models. Meanwhile, although ARDS is defined as 
a syndrome of similar clinical signs associated with damage to the alveolar-capillary membrane, heterogeneity in its etiology has been increasingly recognized. ${ }^{2}$

The major risk factor for ALI/ARDS is pneumonia caused by various microorganisms, accounting for nearly $60 \%$ of the cases. ${ }^{3}$ Pathogens such as bacteria evolve rapidly in response to environmental stress for better adaptation and survival. ${ }^{4-6}$ Compared to the type strains or standard strains preserved and studied in the laboratory for decades, clinical isolates of many bacteria currently exhibit significant differences in phenotype, drug resistance and invasiveness. ${ }^{7-9}$ However, the virulence of this "new generation" of bacteria in causing lung injury is unknown and has not been comparatively explored.

According to the report of the China Antimicrobial Surveillance Network (CHINET) 2019 and 2020, 6 species, Klebsiella pneumoniae, Acinetobacter baumannii, Pseudomonas aeruginosa, Staphylococcus aureus, Streptococcus pneumoniae and Escherichia coli, have become the most commonly found bacteria in the lung, accounting for more than $70 \%$ of the nearly 100,000 strains separated from clinical respiratory specimens nationwide. Are these pathogenic bacteria equally virulent? This has led us to hypothesize that some bacteria may be more harmful in the lung than others, leading to ALI or even ARDS. As a member of CHINET, ${ }^{10}$ the bacterial prevalence in our hospital is a representative part of the status in China. Thus, we isolated the 6 major bacterial species stated above and Streptococcus agalactiae (commonly found in the neonatal ICU) from daily sputum samples. Since the most relevant features of ALI/ ARDS in animal models are rapid onset (within $24 \mathrm{~h}$ ) and histological evidence of tissue injury, ${ }^{11}$ our research has focused on the pathological manifestation of the lungs of mice within $24 \mathrm{~h}$ post treatment. To better visualize the damage, we applied a unilateral lung injury model by inoculating bacteria specifically into the left lung of the animal while keeping their right lungs intact as the control. This method relieved dyspnea, allowed mice to survive until the intended time and avoided systemic influences such as cytokine storm.

\section{Materials and Methods} Ethical Statement

All experiments referring to the use of animals in this study were approved by the Institutional Animal Care and Use Committee of Shanghai Xinhua Hospital affiliated to Shanghai Jiao Tong University School of Medicine (XHEC-F-2018-047). All animal protocols were conducted in compliance with the National Institutes of Health Guide for Care and Use of Laboratory Animals. Efforts were made to minimize the number of animals used and their suffering.

\section{Animals}

Pathogen-free C57BL/6 mice (female, 10 weeks old, $21 \pm 1$ g) and SD rats (female, 6-8 weeks old, 200 \pm 10 g) were purchased from Sippr-BK Laboratory Animal Co. Ltd, Shanghai. All animals were accommodated at the Model Animal Research Center of Xinhua Hospital in a specific pathogen-free animal facility under constant temperature and humidity, with sufficient qualified food and water for 1 week before use.

\section{Unilateral Intratracheal Instillation}

In mice, we selected the left lung for bacterial or exoproducts administration. Meanwhile, the right lung was set as the control lung within each individual. Unilateral lung intubation and instillation are described in the Supplemental Information.

\section{Lung Histopathology}

Mice were sacrificed $24 \mathrm{~h}$ post infection. The lung was inflated with $4 \%$ paraformaldehyde fixative under constant pressure of 15 to $25 \mathrm{~cm} \mathrm{H}_{2} \mathrm{O}$ via the trachea. ${ }^{12}$ The lung was embedded in paraffin block for tissue sections $(5 \mu \mathrm{m})$, and hematoxylin and eosin (HE) staining or TUNEL staining was subsequently performed. The ALI score was performed as described. ${ }^{11}$ In the modified ALI score, we substituted the item "Neutrophils in the interstitial space" with "Red blood cells in the alveolar space" to better differentiate the severity of lung injury resulting from $P$. aeruginosa exoproducts.

\section{Terminal Deoxynucleotidyl Transferase-dUTP Nick-End Labeling (TUNEL) Analysis}

Cellular apoptosis in the lung tissues was detected using a TUNEL staining kit (Roche, Switzerland) according to the manufacturer's instructions. Briefly, the paraffin sections were dewaxed to hydration. Proteinase $\mathrm{K}$ liquid was added to incubate the sections for $30 \mathrm{mins}$ at $37^{\circ} \mathrm{C}$ and then washed away. TUNEL reagent (TdT: $\mathrm{dUTP}=2: 29$, mixed) was added and incubated with the sections for 120 minutes at $37^{\circ} \mathrm{C}$. 
The sections were washed with PBS and subsequently counterstained with DAPI. The stained sections were observed and evaluated using an Olympus IX 70 inverted fluorescence microscope (Olympus, Japan).

\section{Clinical Isolates of Major Bacteria Species}

Between April and July 2020, 7 major bacterial species were nonspecifically separated from the clinical sputum specimen (part of the routine hospital laboratory procedure): Acinetobacter baumannii, Klebsiella pneumoniae, Pseudomonas aeruginosa, Escherichia coli, Staphylococcus aureus, Streptococcus pneumoniae and Streptococcus agalactiae were isolated according to morphology, confirmed by mass spectrometry and stored at $-70^{\circ} \mathrm{C}$. Details of the enrichment culture are described in the Supplemental Information.

\section{P. aeruginosa Strains and Exoproducts}

A total of $8 P$. aeruginosa strains were isolated from 8 clinical respiratory tract specimens between August and September 2020. Pa 1-6 was named according to the separation time. A mucoid strain was named $\mathrm{Pa} \mathrm{M}$, and a strain used in earlier experiments was $\mathrm{Pa} \mathrm{J}$ (isolated in July). The growth medium for routine harvesting of P. aeruginosa exoproducts was Columbia agar supplied with $5 \%$ sheep blood ( $90 \mathrm{~mm}$, Comagal, Shanghai). The entire agar surface was inoculated with a cotton swab. Plates were incubated at $36 \pm 1^{\circ} \mathrm{C}$ for 3 days. Bacteria and exoproducts on the plate were dissolved in sterile NS ( $5 \mathrm{~mL}$ per plate), scratched off and collected. Bacterial cells were removed after centrifugation at $3200 \times \mathrm{g}$ for $30 \mathrm{~min}$ and further cleared by $0.22 \mu \mathrm{m}$ filters. Then, the exoproducts acquired were concentrated by ultracentrifugal filters (10 kDa cutoff value, Amicon Ultra, Merck) at $3000 \times \mathrm{g}$ for $30 \mathrm{~min}$. The protein level of the exoproducts was measured through the BCA method. All exoproducts were adjusted to the same protein concentration by adding sterile normal saline (NS).

\section{PCR and MLST}

Virulence genes were detected by PCR. The primer sequences used are listed in Table S4, and details and results are given in the Supplemental Information.

\section{Gelatin Zymography}

Gelatin zymography was performed as reported by Kessler et al. ${ }^{13}$ Details are described in the Supplemental Information.
Fibrinogenolytic and Fibrinolytic Activities and Thrombin Degradation Assay

Proteolysis activity was tested on bovine fibrinogen, fibrin and thrombin. Details are given in the Supplemental Information.

\section{Protease Assays}

P. aeruginosa exoproducts and purified LasB were tested for elastase activity using the elastin-Congo red (Sigma, US) method. ${ }^{13}$ For exoproducts inhibition assay, protease inhibitors cocktail (PIR) composed of $17 \mathrm{mM}$ AEBSF, $2.5 \mu \mathrm{M}$ Aprotinin, $1 \mathrm{mM}$ Bestatin, $0.1 \mathrm{mM}$ E64, $0.1 \mathrm{mM}$ Leupeptin in DMSO with $8.5 \mathrm{mM}$ EDTA added (Beyotime Biotechnology, Hangzhou) was used to preincubated with $1 \mathrm{mg} / \mathrm{mL}$ exoproducts at room temperature for $30 \mathrm{~min}$.

\section{Proteomic Analysis of Exoproducts}

Exoproducts from different strains were separated by $10 \%$ polyacrylamide gel electrophoresis (SDS-PAGE) and stained with Coomassie blue. The whole lane or certain protein bands of interest were excised from the gel and processed by mass spectrometry (LTQ Orbitrap Velos Pro, Thermo Finnigan) coupled to liquid chromatography as previously described. ${ }^{14}$ Mascot 2.3 software was used for data analysis.

\section{Purification of LasB}

The protease was purified from exoproducts by ammonium sulfate precipitation followed by DEAE-cellulose chromatography. Further details are given in the Supplemental Information.

\section{Cytotoxicity Assay}

THP-1 cells (TIB 202, ATCC) were seeded in 96-well plates with 1640 medium containing $10 \%$ fetal bovine serum. Purified LasB at a concentration of $100 \mu \mathrm{g} / \mathrm{mL}$, $\mathrm{Pa} 4$ exoproducts at a concentration of $200 \mu \mathrm{g} / \mathrm{mL}$ or $\mathrm{Pa} 4$ bacteria at a concentration of $7.5 \times 10^{7} \mathrm{CFU} / \mathrm{mL}$ were added to the wells. Cytotoxicity was measured by LDH release 4 hours post incubation using LDH cytotoxicity assay kits (Beyotime Biotechnology, Hangzhou) according to the manufacturer's instructions.

\section{Hemostasis Function}

To examine the effect on hemostasis function, $20 \mu \mathrm{g}$ LasB was added to $2 \mathrm{~mL}$ blood ( $1.8 \mathrm{~mL}$ blood $+0.2 \mathrm{~mL}$ sodium citrate anticoagulant) driven from healthy volunteers and 
incubated for $35 \mathrm{~min}$ at room temperature. The samples were then subjected to automatic hemostasis tests by ACL TOP (550 CTS, Werfen, US).

\section{Statistics}

The data are expressed as the mean \pm standard error of the mean. Statistical analysis was performed in GraphPad Prism 8 software using one-way or two-way t-tests or $\log$ rank tests as appropriate. Statistical significance is indicated as $(* \mathrm{P}<0.05 ; * * \mathrm{P}<0.005 ; * * * \mathrm{P}<0.0005)$. Data are representative of at least three independent experiments.

\section{Results}

\section{Mice Model of Unilateral Lung Injury}

We used a unilateral lung injury model in this study through tracheotomy and intubation into the left lung of the mice. We verified the method through LPS instillation. CT reconstruction (PINGSHENG, Shanghai) in Figure 1A shows that instillation of $30 \mu \mathrm{L}$ of $5 \mathrm{mg} / \mathrm{mL}$ LPS successfully induced consolidation of the whole left lung in 4 hours.

This animal model was initially designed to see if heavy unilateral lung injury would provoke bilateral alveolar damage through systemic impacts such as cytokine storm, whereas we could find no such phenomenon in mice tested with lipopolysaccharide, acid, porcine elastase or mechanical ventilation, even if severe inflammation and animal death were observed (unpublished data). The untreated lungs of unilaterally injured mice exhibited no difference from the lungs of intact normal mice (Figure S1A).

\section{Clinically Isolated Gram-Negative Bacteria Induced More Severe Lung Injury Than Gram-Positive Bacteria}

We first tested the major gram-negative bacterial species, which together accounted for $60 \%$ of all clinical respiratory isolates. Mice were instilled with $25 \mu \mathrm{L}$ bacterial suspensions $\left(2.25 \times 10^{7} \mathrm{CFU}\right)$ of Klebsiella pneumoniae, Acinetobacter baumannii, Escherichia coli or Pseudomonas aeruginosa. As shown in Figure 1B, alveolar injury occurred in all groups within $24 \mathrm{~h}$. Lung tissue injury caused by $K$. pneumoniae was noted because a large number of leukocytes accumulated in the interstitial tissue, while the alveolar space was filled with mucus. However, the mucus was later proven to be hypermucoviscous $K$. pneumoniae bacteria instead of protein debris or edema from the hosts (Figure S1C). Lungs infected with A. baumannii and $E$. coli both triggered neutrophil accumulation, which was patchy in the A. baumannii group but more diffused in the $E$. coli group. However, no hyaline membrane, red blood cell effusion or septal thickening was found in either group. Strikingly, the 3 independently isolated $P$. aeruginosa strains all caused hemorrhage, severe hyaline membrane formation, neutrophilic infiltration, parenchymal edema and proteinaceous debris deposits in the infected lungs, fitting the histological change of DAD (Figure 1B).

Next, to study the lung injury effect of gram-positive bacteria in vivo, $2.25 \times 10^{7} \mathrm{CFU}$ of $S$. aureus, $S$. agalactiae or $S$. pneumoniae suspended in $25 \mu \mathrm{L}$ PBS was instilled into the left lung of mice. Much to our surprise, all mouse groups remained healthy $24 \mathrm{~h}$ post inoculation. Lung histology found no obvious alveolar damage (Figure 1C). Considering that these daily isolated gram-positive bacteria may have relatively low virulence, we further tested a hypervirulent mucoid serotype $3 \mathrm{~S}$. pneumoniae stored earlier, of which we had to reduce the bacteria number to $2.25 \times 10^{5} \mathrm{CFU}$ to keep half of the inoculated mice live to the $24 \mathrm{~h}$ endpoint. However, no visible alveolar damage occurred, even though $50 \%$ of mice died or developed sepsis and multiple organ infection within $24 \mathrm{~h}$ (Figure S1B). A large number of neutrophils were found, but the accumulation was limited to the perivascular space of lung arteries (Figure 1C). These results showed that irrelevant to their virulence, the major gram-positive bacteria tested evoked bronchial pneumonia-like lung injury rather than the DAD found in ALI/ARDS.

We then compared the ALI score caused by each species (Figure 1D). Generally, gram-negative bacteria achieved much higher scores than gram-positive bacteria. $P$. aeruginosa acquired the highest score, indicating its great potence in ALI/ARDS pathogenesis. Thus, we isolated more strains of $P$. aeruginosa to analyze their injury mechanism. The baseline characteristics of the patients from whom the strains were separated are provided in Table 1. The strains are different in morphology (Figure S3) and have different multilocus sequence typing profile (Table S3).

\section{Hypervirulent $P$. aeruginosa Strains Infected Unilaterally Leads to Bilateral DAD}

Eight $P$. aeruginosa strains, $\mathrm{Pa} 1-6, \mathrm{~Pa} \mathrm{M}$ and $\mathrm{Pa} \mathrm{J}$, were stilled unilaterally in mice, and survival curves were established. Quality control strain ATCC27853 was used as a comparison. As shown in Figure 2A, $\mathrm{Pa} 4,5$, and 6 are the most pernicious. $\mathrm{Pa} 3$ was the least virulent among clinical isolates, while ATCC27853 was the least virulent 
A

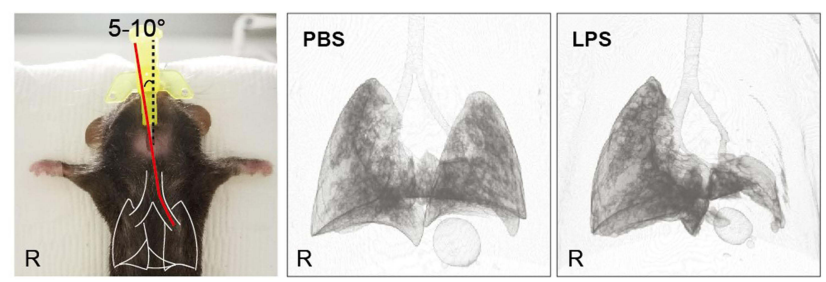

B

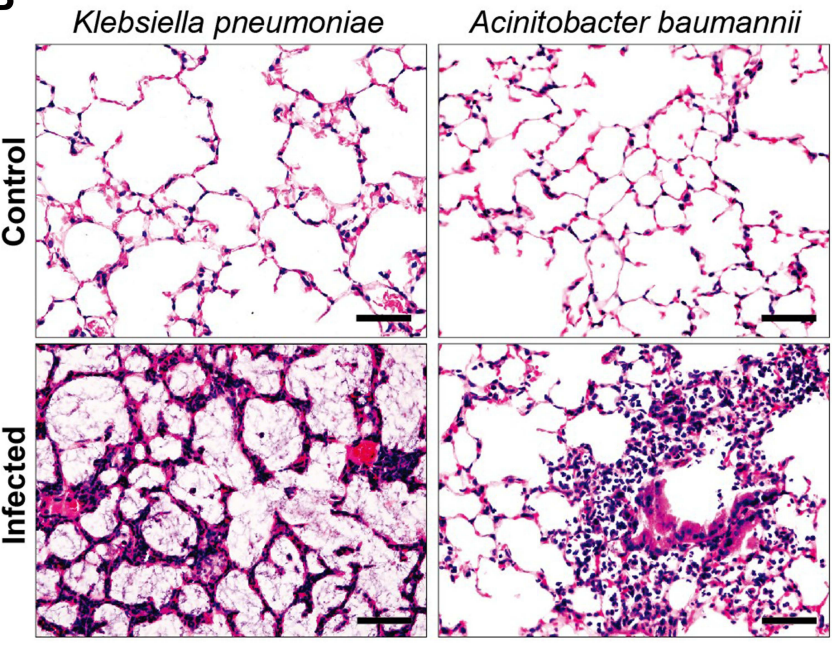

D

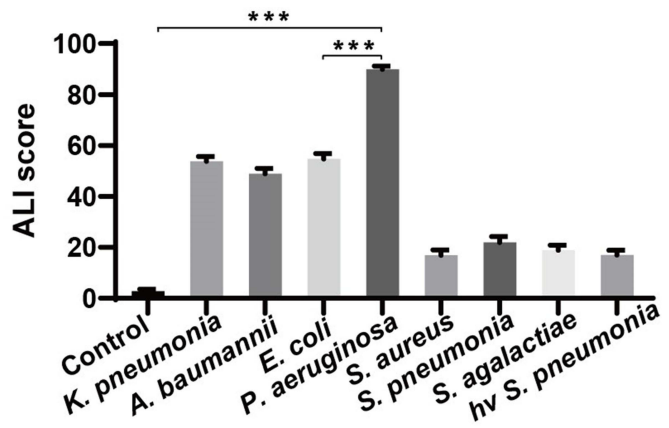

Escherichia coli
Pseudomonas aeruginosa

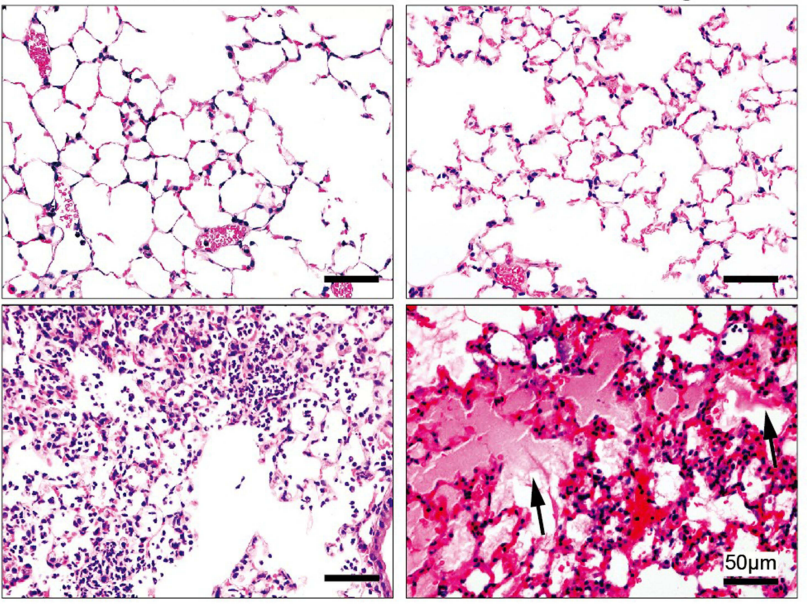

C

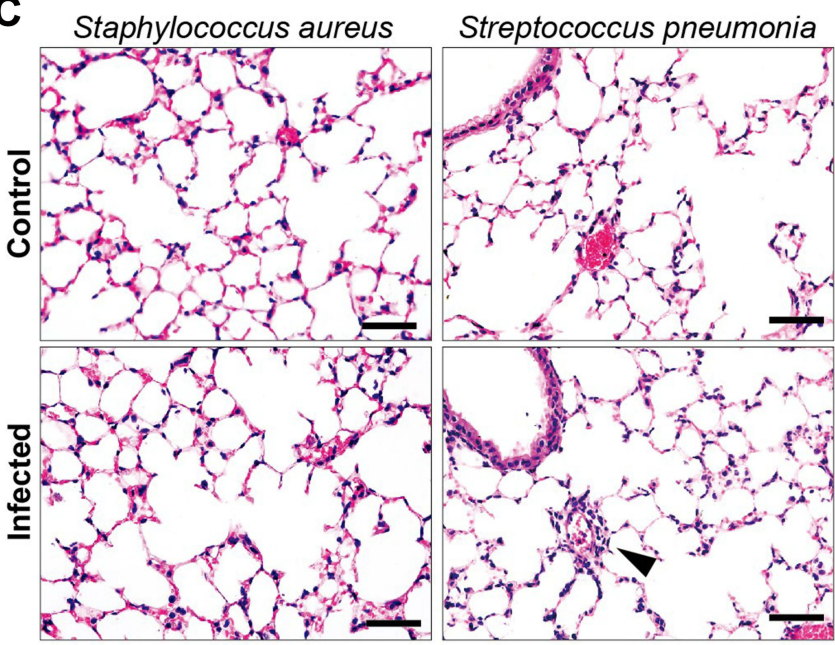

Streptococcus agalactiae hv Streptococcus pneumonia

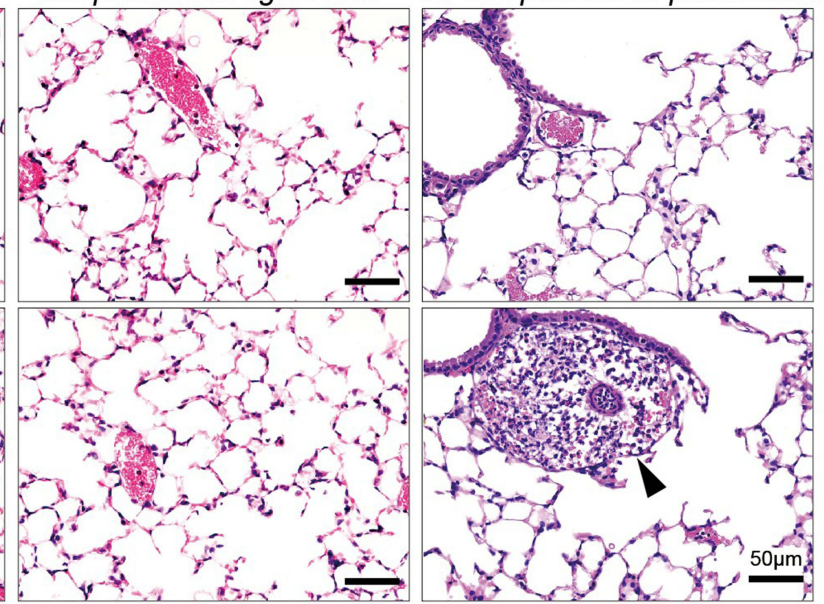

Figure I Histological assessment of acute lung injury induced by major bacterial species separated from clinical sputum (A) Unilateral endotracheal intubation. Unilateral lung consolidation appeared 4 hours after LPS instillation (3D CT reconstruction). (B) Hematoxylin and eosin (HE) stain of lung sections inoculated with gram-negative bacteria. Alveolar injury was observed in all groups ( $\mathrm{n}=9$ in each group). $K$. pneumoniae: neutrophils were accumulated in the alveolar wall and the alveolar space was filled with bacteria; $A$. baumannii: patchy neutrophil infiltration; $E$. coli: diffuse neutrophil infiltration; $P$. aeruginosa: hemorrhage, diffuse alveolar damage, hyaline membranes (arrows), vessel congestion, alveolar wall thickening and neutrophil infiltration were all observed. (C) HE stain of lung sections of gram-positive bacterial infection. Neutrophil infiltration was found limited in the periarterial space (arrowheads) without causing alveolar damage, especially in hypervirulent (hv) Streptococcus pneumonia group ( $\mathrm{n}=9$ in each group). (D) Score of 7 major bacterial species induced ALI 24h post-inoculation in mice, compared with normal C57/BL6 mice ( $\mathrm{n}=6$ per group). *** $\mathrm{P}<0.0005$.

in all strains. Pa 2 was less toxic than $\mathrm{Pa} 4,5$, and 6 $(\mathrm{p}=0.003)$ but more toxic than $\mathrm{Pa} 1,3, \mathrm{M}$ and $\mathrm{J}(\mathrm{p}<0.0001)$.

Mice instilled with $\mathrm{Pa} 2$ and $\mathrm{Pa}$ 4, 5, and 6 all died within $24 \mathrm{~h}$. They also achieved higher ALI score (Figure 2B).
Meanwhile, pink frothy sputum and bloody sputum which resemble the symptoms of ALI/ARDS patients, were seen from the nose and mouth in 50\% of the animals (Figure 2E and F). Autopsy found that $\mathrm{Pa} 4,5$, and 6 caused a similar pathological change, prominent with lung hemorrhage, 
Table I Baseline Characteristics of Patients from Whom the Strains Were Isolated

\begin{tabular}{|l|c|c|c|c|c|c|c|c|}
\hline & Pa I & Pa 2 & Pa 3 & Pa 4 & Pa 5 & Pa 6 & Pa M & Pa J \\
\hline Age & 83 y & $28 d$ & $2 y$ & $35 y$ & 53 y & 76 y & 79 y & 50 y \\
Sex & M & M & F & M & M & F & M & M \\
ICU stay & No & Yes & Yes & Yes & Yes & Yes & No & No \\
Clinical diagnosis & Pneu & Pneu & ARDS & Pneu & Pneu & ARDS & AEC & Pneu \\
\hline
\end{tabular}

Abbreviations: $y$, year; d, day; M, male; F, female; Pneu, pneumonia; ARDS, acute respiratory distress syndrome; AEC, acute exacerbation of chronic obstructive pulmonary disease.

DAD, neutrophil infiltration and septal thickening (Figure 2D). To our surprise, $87 \%$ of mice infected unilaterally with these 3 strains developed bilateral lung injury (Figure 2C), which is an important clinical feature of ARDS patients but was poorly recapitulated in animals before. ${ }^{15}$ However, in the P. aeruginosa uninoculated lungs, DAD was mostly observed around the bronchus or bronchia instead of the alveoli, indicating that it was likely caused by aspiration of the bloody sputum or bacteria from the infected lung instead of systemic influence.

\section{Exoproducts from $P$. aeruginosa} Reproduced Hemorrhagic DAD Caused by Bacterial Infection

We examined the virulence genes (Table S1), drugresistance and pyocyanin production of all 8 P. aeruginosa strains (Table S2), however, they did not show a clear relation with the virulence. Since all 8 P. aeruginosa strains tested were positive for the T1SS and T2SS genes but not the T3SS genes, we collected their extracellular products and examined virulence in vivo and
A
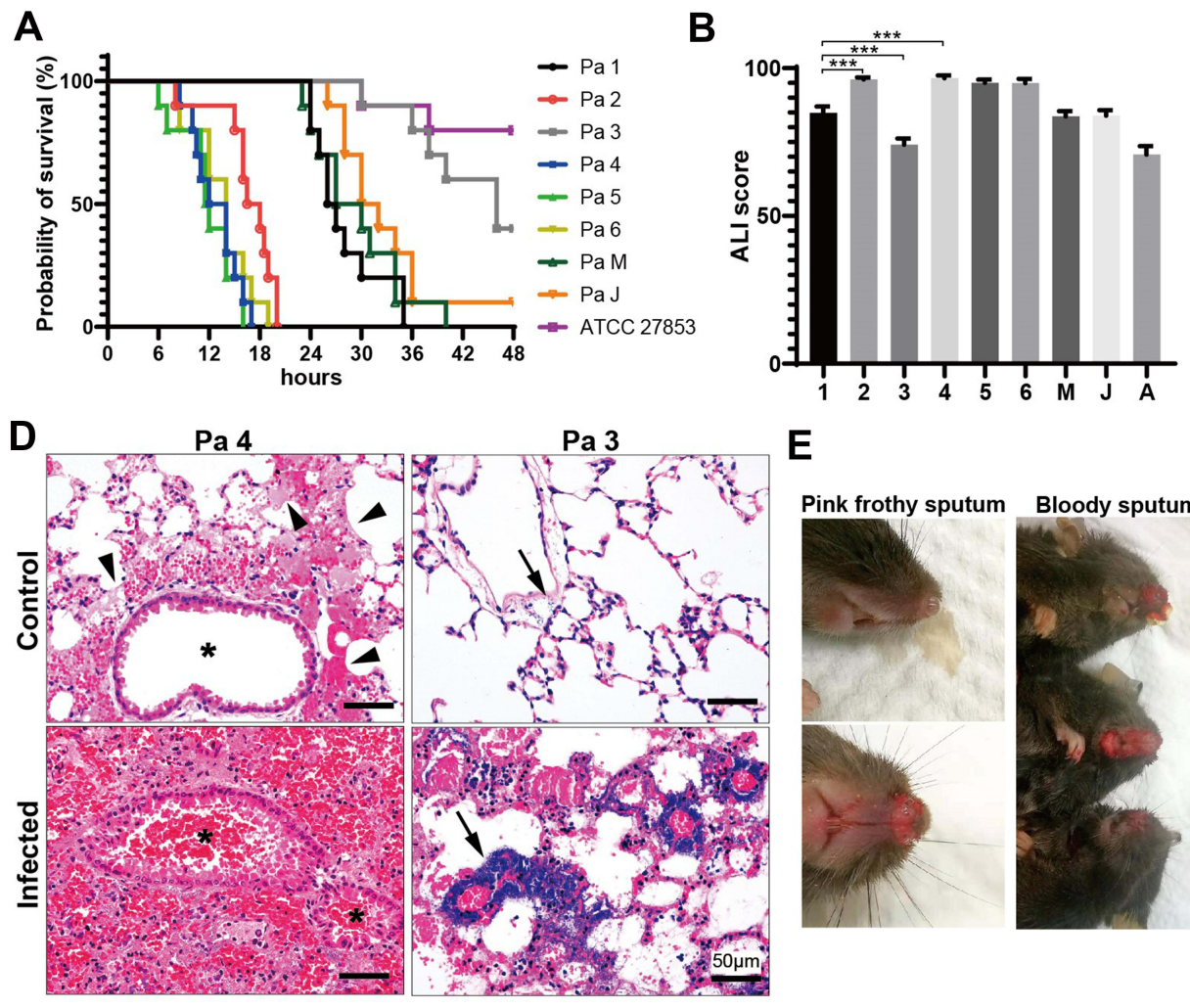

E

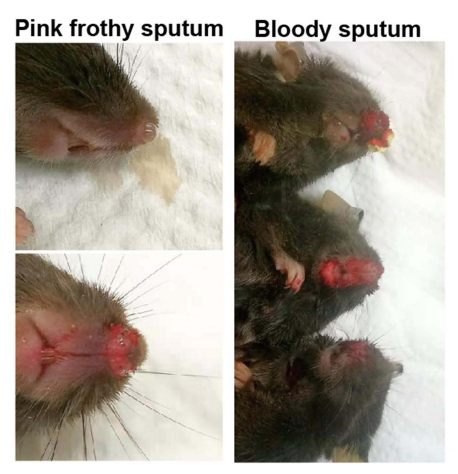

C

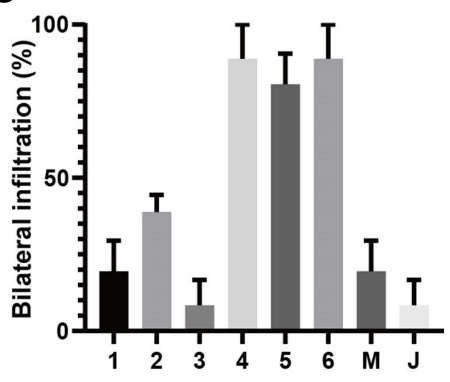

$\mathbf{F}$

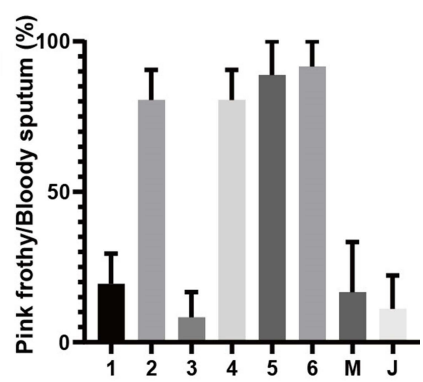

Figure 2 ALI/ARDS induced by different strains of $P$. aeruginosa in mice (A) Kaplan-Meier survival curves for mice infected with different strains ( $\mathrm{n}=10$ in each group). (B) ALI score of mice infected with different strains $(n \geq 4)$. Mice were euthanized at $12 \mathrm{~h}$ post infection. A, ATCC27853; *** $<<0.0005$. (C) Rate of occurrence of bilateral infiltration $12 \mathrm{~h}$ after unilateral inoculation ( $\mathrm{n}=10$ in each group). (D) Representative histology findings of the most and least virulent clinical $P$. aeruginosa strain Pa 4 and $P a 3$. Severe DAD and bilateral infiltration occurred in Pa 4 infected lungs. The bronchial lumen (*) was inundated with red blood cells. DAD, hyaline membranes (arrowheads) and neutrophil accumulation even occurred in the uninoculated control lung in $\mathrm{Pa} 4$ infected mice; In Pa 3 infected group, less hemorrhage but more obvious bacterial proliferation and infiltration found in bilateral lungs (arrows). HE stain; (E) pink frothy sputum and bloody sputum observed from the nose and mouth of mice. (F) Rate of occurrence of pink frothy/bloody sputum in different mice groups ( $\mathrm{n}=10$ per group). 
in vitro. As shown in Figure 3A, the exoproducts (1 mg protein $/ \mathrm{mL}$ ) reproduced the typical histologic change of $P$. aeruginosa lung infection. The gross appearance of the left lungs showed different severities of injury, which was largely alleviated by proteinase inhibitor cocktail with EDTA added. The modified ALI score clearly shows that exoproducts from $\mathrm{Pa} 4,5$, and 6 are the strongest in inducing ALI, while $\mathrm{Pa} 3$ is the weakest (Figure 3C). This tendency is consistent with the virulence of the corresponding bacteria (Figure 2A). To our surprise, when the concentration of the exoproducts was elevated to $4 \mathrm{mg}$ protein $/ \mathrm{mL}$, it not only caused severe hemorrhage and DAD in all animal groups ( $\mathrm{n}=3$ mice, 8 group) but also the death of 14 mice in merely 3 hours. Autopsy found tension pneumothorax and lung compression in these mice. In contrast, surviving mice showed emphysematous alveolar destruction without lung consolidation (Figure 3B).

Interestingly, exoproducts lost the ability to cause alveolar hemorrhage or hyaline membranes after heating at $100^{\circ} \mathrm{C}$ for 10 mins (Figure S2A). We then found that the extracted bacterial composition of $P$. aeruginosa could not provoke hemorrhage or hyaline membranes either (Figure $\underline{\mathrm{S} 2 \mathrm{~B}}$ ). These findings suggest that $P$. aeruginosa mainly injured the lung through heat labile extracellular products, which were most likely proteases instead of heat stable substances, such as DNA or LPS.

$P$. aeruginosa induced cell apoptosis is the most widely studied cell death in ALI/ARDS. ${ }^{16}$ To determine whether and which pulmonary cell apoptosis was provoked, TUNEL labeling was used to stain $P$. aeruginosa bacteria or exoproducts injured lung tissue (Figure 3D and E). To our surprise, although both caused severe DAD, the exoproducts only induced apoptosis of $5.19 \%$ pulmonary cells compared to the $71.7 \%$ apoptosis rate by live bacteria. This result revealed that cell damage was present but probably not essential for the appearance of DAD in $P$. aeruginosa infection.

Consequently, we infer that hemorrhagic lung injury may have resulted from the destruction of extracellular matrix and disturbance of hemostasis. Collagen and elastin are the major protein components of the pulmonary extracellular matrix and are essential for physiological function and the initiation of blood coagulation. First, we applied gelatin zymography to visualize the degradation of collagen by exoproducts (Figure 3F). All exoproducts from $P$. aeruginosa exhibited multiple bright bands on zymography, especially hypervirulent strains $\mathrm{Pa} 4,5$ and 6. Most exoproducts showed a major band at the same level at $120 \mathrm{kDa}$, while $\mathrm{Pa} 2$ manifested as a major band near $80 \mathrm{kDa}$. The activity was largely inhibited by EDTA. We then measured the elastin lytic activity of each exoproducts using the elastin-Congo red method (Table 2). Exoproducts exhibited different elastinolytic activities. The level has a tendency to be positively related to the ALI score of exoproducts and the virulence of the corresponding $P$. aeruginosa strains. Apparently, exoproducts from $P$. aeruginosa are powerful in destroying pulmonary structural compositions, especially $\mathrm{Pa} 4,5$ and 6 .

As fibrinogen and thrombin are the two most important protein factors in coagulation, their degradation could have contributed to hemorrhagic lung injury. Thus, we examined the proteolytic ability of exoproducts. As shown in Figure $3 \mathrm{G}$, all 8 exoproducts from $P$. aeruginosa strains hydrolyzed fibrinogen efficiently. We used several protease inhibitors and found that fibrinogen lysis was most significantly suppressed by the MMP inhibitor Ilomastat. Freshly formed fibrin clots were digested by all exoproducts as well (data not shown). The exoproducts also exhibited a strong ability to hydrolyze thrombin, as shown in the thrombin degradation assay (Figure $3 \mathrm{H}$ ). The effect was partially inhibited by EDTA (EDTA usage was much larger due to the $\mathrm{Ca}^{2+}$ ions contained in commercially acquired thrombin). These findings suggest that virulence effectors in the exoproducts of $P$. aeruginosa are most likely matrix metalloproteases.

\section{Proteomic Analysis of Exoproducts and Purification of the Virulent Protease LasB}

$P$. aeruginosa exoproducts were then subjected to SDSPAGE to analyze the secretory proteome (Figure 4A). LCMS/MS analysis found that the most abundant protein secreted by $\mathrm{Pa} 4,5$ and 6 at $35 \mathrm{kDa}$ is LasB elastase. Relative LasB expression in the exoproducts of 8 $P$. aeruginosa strains fits their lung damage ability (Figure 4B). The intense band appearing at a molecular mass of $70 \mathrm{kDa}$ from less virulent strains also caught our attention, which, however, was found to be catalase or acetylated catalase. Meanwhile, no LasA was detected from $\mathrm{Pa} 2$ or $\mathrm{Pa} 4$ exoproducts.

Taken together, the results of LC-MS/MS and gelatin zymography indicated that strain $\mathrm{Pa} 4$ could be used to culture and harvest LasB. LasB was separated from the exoproducts of $\mathrm{Pa} 4$ by DEAE-cellulose chromatography. 
A
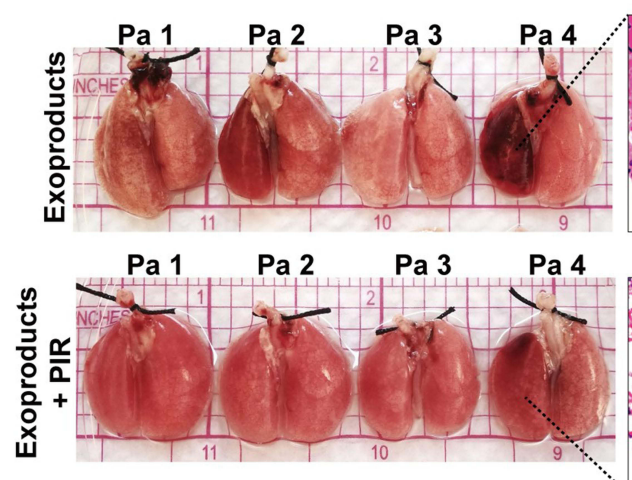

C

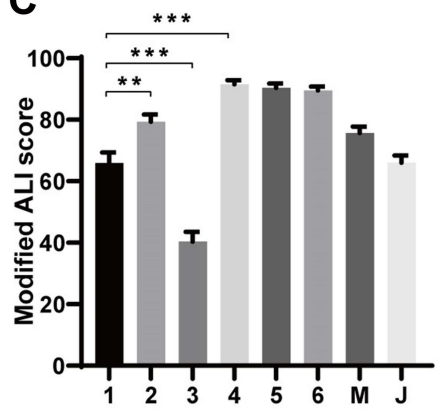

F

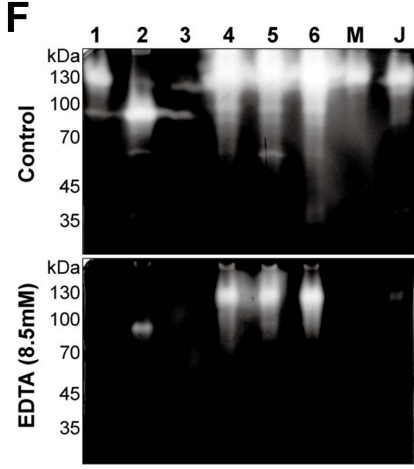

D

G
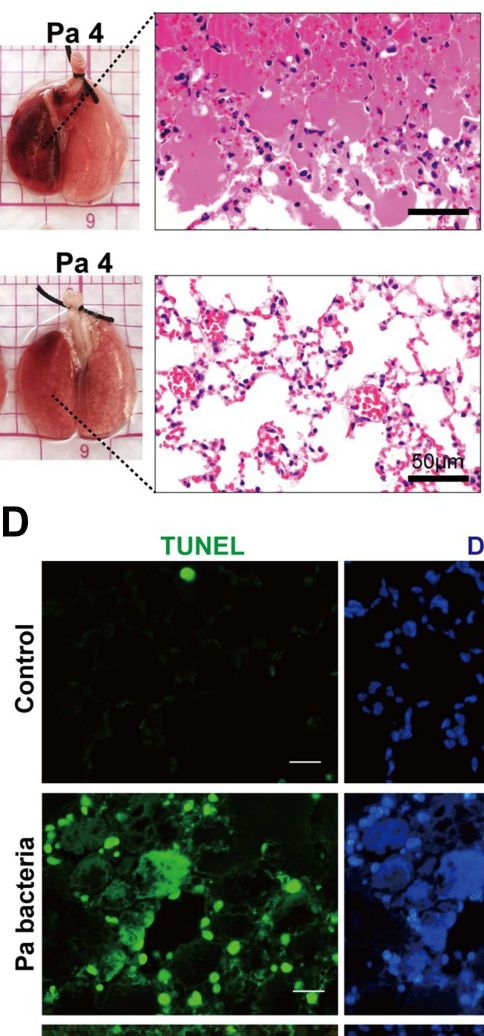

B No pneumothorax

Pneumothorax

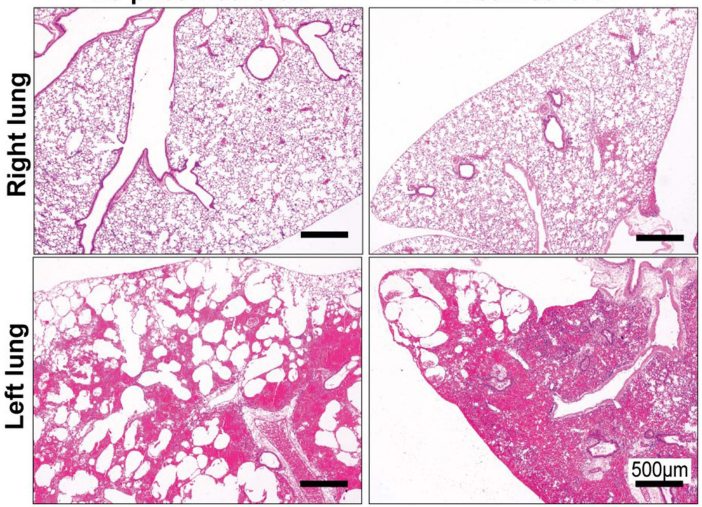

soling
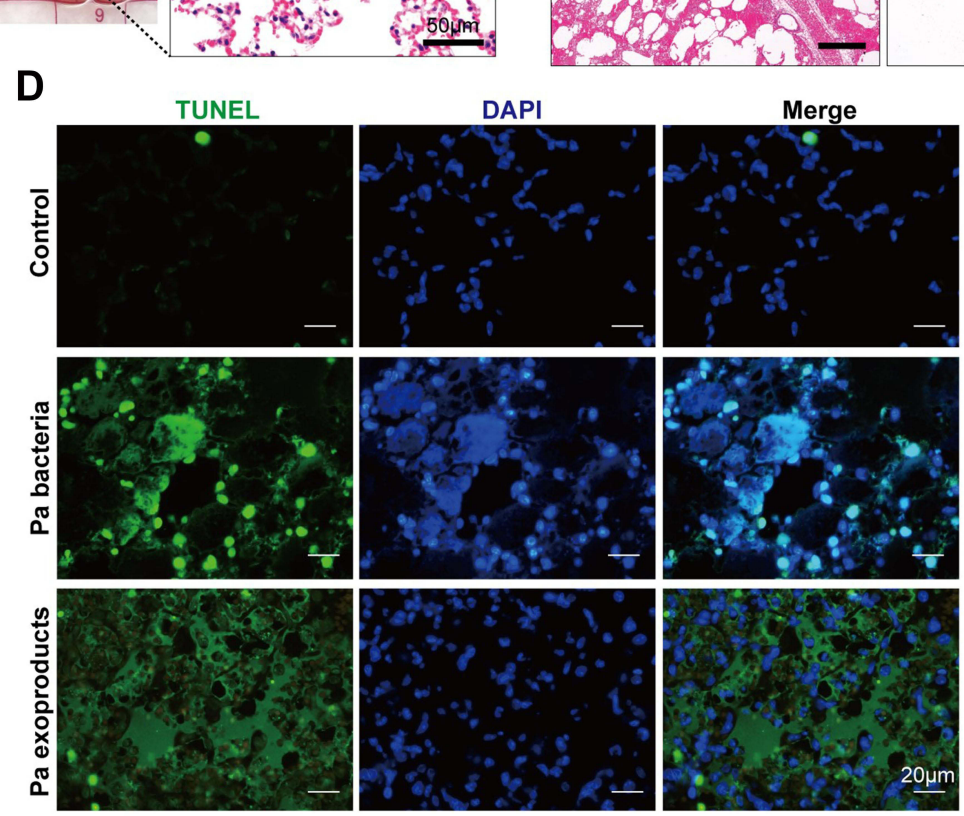

E
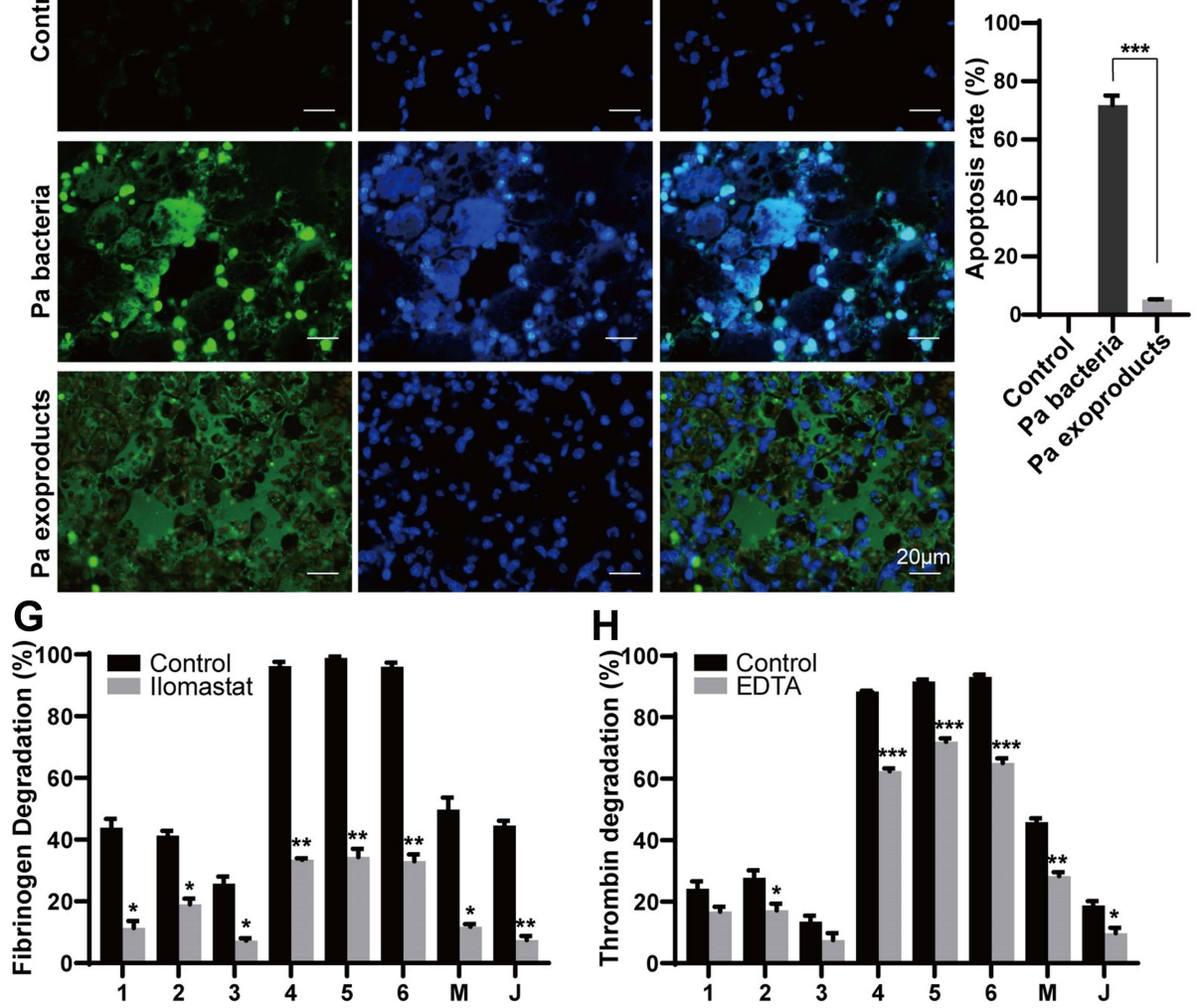

Figure $3 P$. aeruginosa exoproducts induce hemorrhagic ALI in mice. (A) Representative gross picture of $P$. aeruginosa exoproducts injured mice lung. Unilateral lung injury was largely inhibited by PIR (protease inhibitors cocktail with EDTA added). Typical DAD, hyaline membranes and bleeding were induced by Pa 4 exoproducts. (B) Histological difference of mice died with pneumothorax or not $3 \mathrm{~h}$ after high concentration of exoproducts instillation. Severe lung tissue compression appeared in mice died of pneumothorax $(n=14)$, compared with emphysematous alveolar destruction in survived mice (no pneumothorax, $n=10)$. (C) Modified ALI score of different $P$. aeruginosa strain exoproducts challenged lungs. $n=3$. (D) TUNEL stain of $P$. aeruginosa bacteria/exoproducts challenged lung tissue. Cell nuclei (Blue), TUNEL-positive cells (Green). (E) Apoptosis rate of bacteria/exoproducts challenged lung cells. (F) Gelatin zymography of exoproducts. (G) Fibrinogen degradation assay of exoproducts. (H) Thrombin degradation assay of exoproducts. ${ }^{*} \mathrm{P}<0.05$; ${ }^{* *} \mathrm{P}<0.005$; $* * * \mathrm{P}<0.0005$.

As shown in the elution profile (Figure 4C), the only active enzyme was eluted between $0.1 \mathrm{M}$ to 0.25 $\mathrm{M} \mathrm{NaCl}$ (fractions 5-15). Fraction 10 was collected and concentrated to $2 \mathrm{mg} / \mathrm{mL}$ protein. The purity of protease was verified by SDS-PAGE as a single band at $35 \mathrm{kDa}$, which was confirmed as LasB elastase (Figure 4D). Purified LasB exhibited an increase in elastase-specific activity (Table 2). As shown previously on zymography (Figure 3), $\mathrm{Pa} 2$ exoproducts presented a clear band at 80 $\mathrm{kDa}$, different from the rest. However, LC-MS/MS 
Table 2 Elastase Activity of Exoproducts Measured by Elastin-Congo Assay

\begin{tabular}{|l|c|c|c|c|c|c|c|c|c|}
\hline & Pa I & Pa 2 & Pa 3 & Pa 4 & Pa 5 & Pa 6 & Pa M & Pa J & Purified LasB \\
\hline Elastase activity (U) & 1.59 & 3.23 & 0.57 & 12.5 & 12.6 & 15.1 & 3.33 & 2.18 & 90.96 \\
\hline
\end{tabular}

analysis found no proteases other than LasB (Figure 4E). Marquart et al reported the same phenomenon. ${ }^{17}$ Some studies point out that this might be due to differences in polymer conformation, since aggregation of LasB monomers to different molecular weight polymer forms is commonly found on gelatin zymograms. ${ }^{18}$

\section{LasB Elastase Provoked Hemorrhagic DAD Through Degradation of the Alveolar Matrix and Key Proteins in the Coagulation Cascade}

Figure 5A presents the histological changes in the lungs of mice after being instilled unilaterally with $3 \mu \mathrm{g}$ LasB for 24 h. Significant DAD, hemorrhage, hyaline membrane and emphysematous alveolar destruction were formed in the left lung. The histopathological change is consistent with the injury caused by $P$. aeruginosa bacteria and exoproducts. Lung injury could be alleviated by EDTA and to a greater extent by MMP inhibitor ilomastat (Figure 5B). Additionally, we found $70 \%$ of the mice died in 3 hours from tension pneumothorax when LasB usage was higher than $6 \mu \mathrm{g}(\mathrm{n}=10)$.

LasB showed high activity on gelatin zymography, which was manifested as a single clear band at a molecular weight of $120 \mathrm{kDa}$, thus proving that it is powerful for cleaving collagen (Figure 5C). Similar to the exoproducts, LasB exhibited a strong capability to hydrolyze fibrinogen and thrombin (Figure 5D). The process was attenuated by both EDTA and ilomastat but not by TLCK. To examine the overall effect of LasB on clotting, we incubated LasB with citrate-anticoagulated healthy human blood. As shown in Figure 5E, LasB drastically reduced fibrinogen levels, increased FDP and D-dimer levels and elongated thrombin time (TT), causing DIClike coagulopathy in vitro. However, it did not change the indicators of the endogenous coagulation pathway, such as APTT and PT, or affected the activity of antithrombin (AT) or plasminogen (PA). We then studied the fibrinogen lysis effect in vivo by injecting LasB intravenously into rats (2 $\mathrm{mg} / \mathrm{kg}$ ). Significant fibrinogen reduction was also observed $1 \mathrm{~h}$ post injection (Figure 5F). However, no difference was detected in thrombin time or other indicators.

Finally, we applied TUNEL staining to look for possible cellular apoptosis. Similar to the exoproducts, no significant difference in pulmonary cell apoptosis was observed between LasB-damaged lungs and intact lungs (Figure 5G and $\mathrm{H}$ ), meaning that LasB has little cytotoxicity toward pulmonary cells in vivo. Additionally, the THP-1 cytotoxicity assay found that purified LasB was not apparently virulent to cells, unlike the bacterium (Figure 5I).

\section{Discussion}

Pneumonia is the major risk factor for ALI/ARDS. In most patients suspected or diagnosed with pneumonia, infection of bacteria is most commonly found, sometimes even secondary to virus or fungus infection. ${ }^{19,20}$ In this study, we examined the capability of 7 major pathogenic bacteria isolated from patients to induce ALI in mice. The 3 major gram-positive bacterial species did not cause obvious alveolar injury. Although $S$. pneumoniae is considered a primary pathogen of ARDS, ${ }^{21}$ we found that it mainly induced inflammation in the periarterial space. In comparison, gram-negative bacteria are much more deleterious. $P$. aeruginosa was found to be the most destructive, rapidly inducing hemorrhagic DAD, pink frothy sputum and bilateral lung infiltration similar to the clinical features of ALI/ARDS patients in animals. In this study, we isolated 8 clinical strains of $P$. aeruginosa that are different in morphology, pigment production, T3SS genes and many aspects. LasB production was the only factor we found that correlated in proportion with virulence in the lung. A high dosage of LasB even caused pneumothorax, bullae formation and death in mice in merely 3 hours.

$P$. aeruginosa is an opportunistic pathogen ubiquitously present in the environment. For more than $4 \mathrm{dec}-$ ades, it has held a nearly unshakable position in the rank order of pathogens causing ICU-related infections. ${ }^{22}$ The correlation of $P$. aeruginosa to high mortality of patients with hospital-acquired and ventilator-associated pneumonia (VAP) in the ICUs has aroused serious concerns. ${ }^{23}$ $P$. aeruginosa are known to have a plethora of virulence 


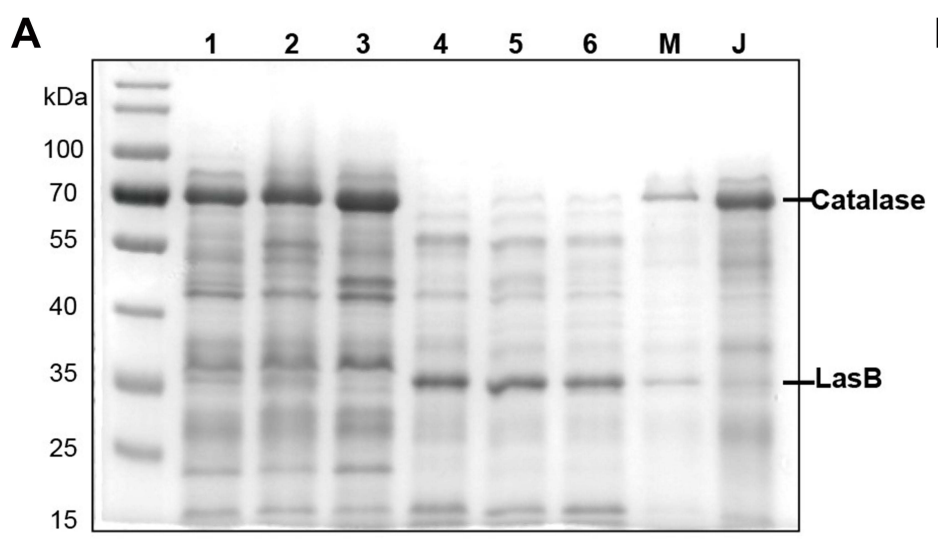

B
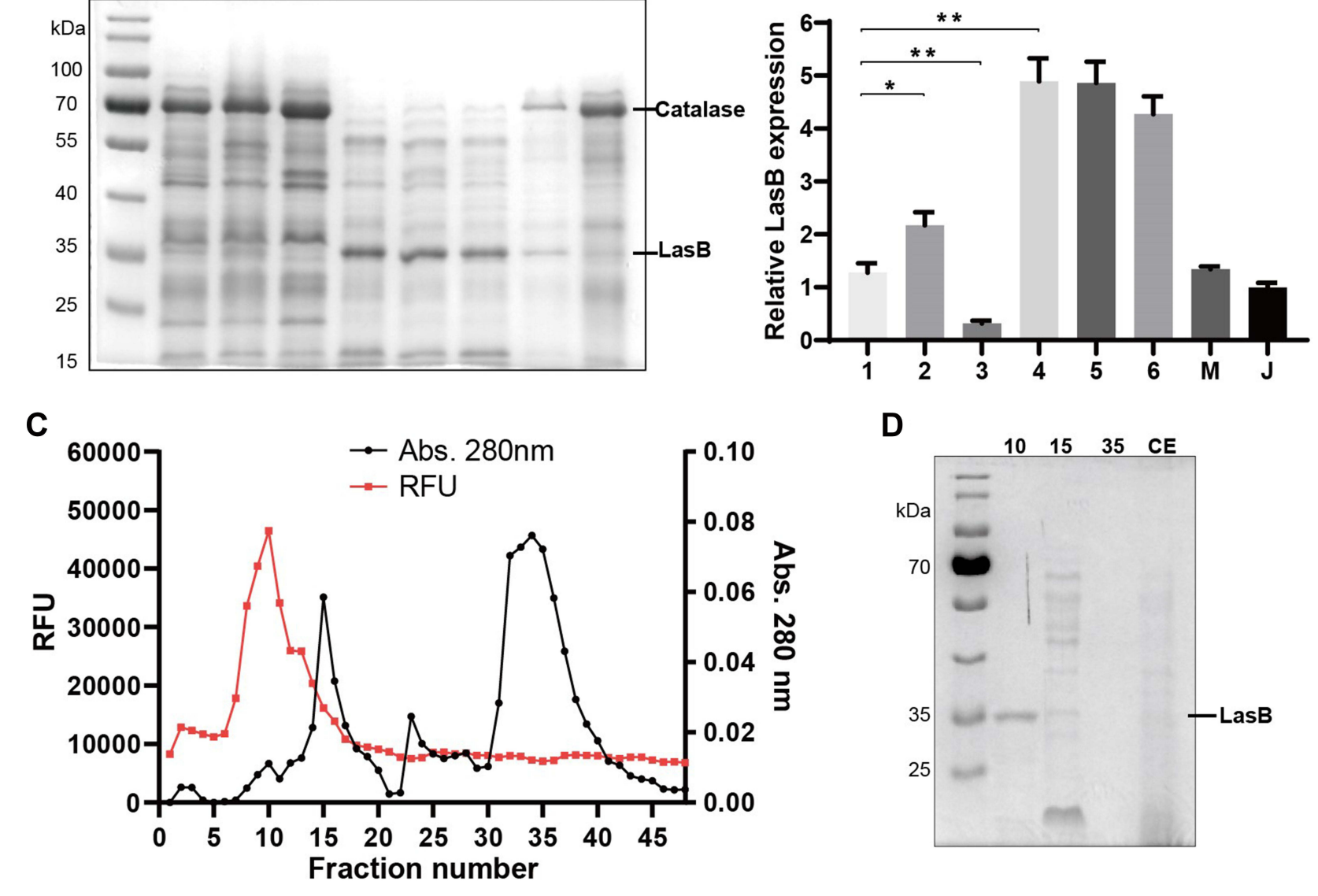

D

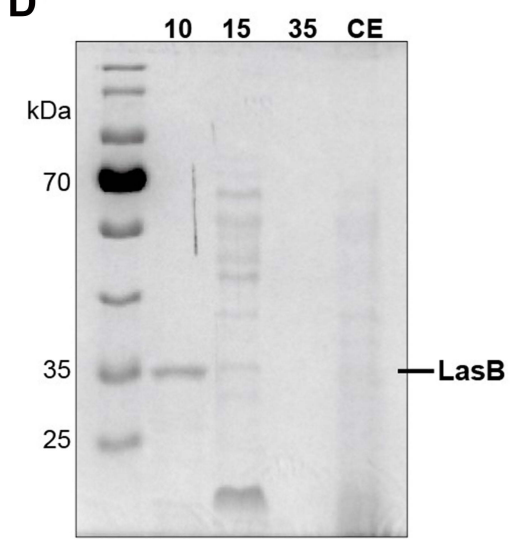

E

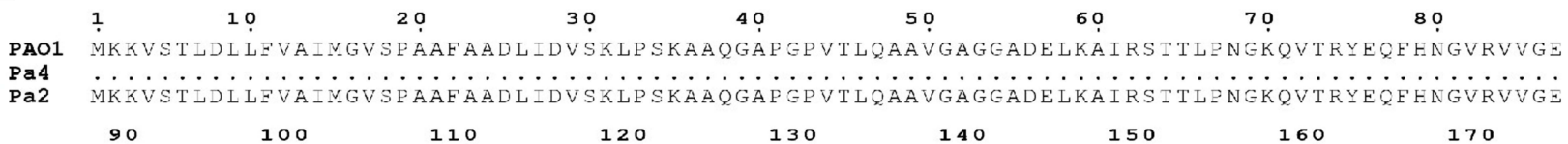

PAO1 AITIVKGPGKSVAAQRSGHFVANIAADIPGSTIAAVSAEQVLAQAKSLKAQGRKIENDKVELVIRLGENNIAQLVYNVSYLIPGEGL

Pa4 AOITEVKGPGKSVAAQRSGHFVANIAADLPGSTIAAVSAEQVZAQAKSLKAQGRKIENDKVELVIRLGENNIAQLVYNVSYLIPGEGL

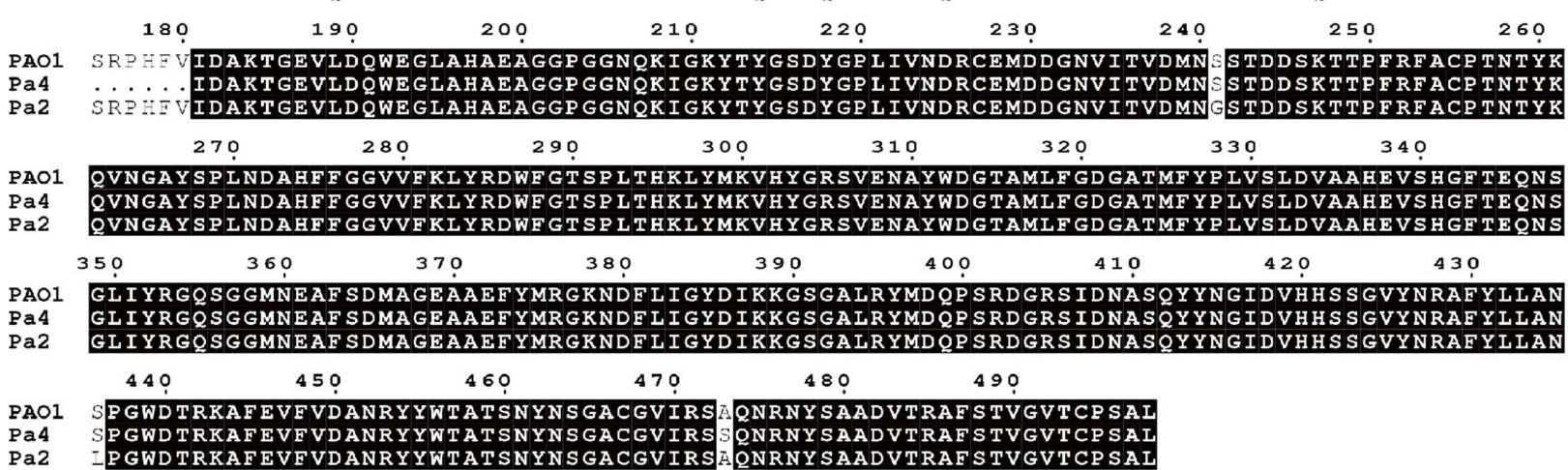

Figure 4 Proteomic analysis of exoproducts and purification of the virulent protease LasB (A) SDS-PAGE of exoproducts from 8 P. aeruginosa strains. (B) Relative LasB expression of 8 P. aeruginosa strains. ${ }^{*} \mathrm{P}<0.05$; ${ }^{* * P}<0.005$; (C) elution profile of LasB protease on DEAE 52 cellulose column. (D) Verification of LasB purity by SDS-PAGE. 10, purified LasB (Fraction 10); 15, impurities (Fraction 15); 35, impurities (Fraction 35); CE, crude enzyme solution. (E) Amino acid sequence alignment of Las B from Pa 4, $\mathrm{Pa} 2$ and PAOI.

Abbreviation: RFU, relative fluorescence unit. 

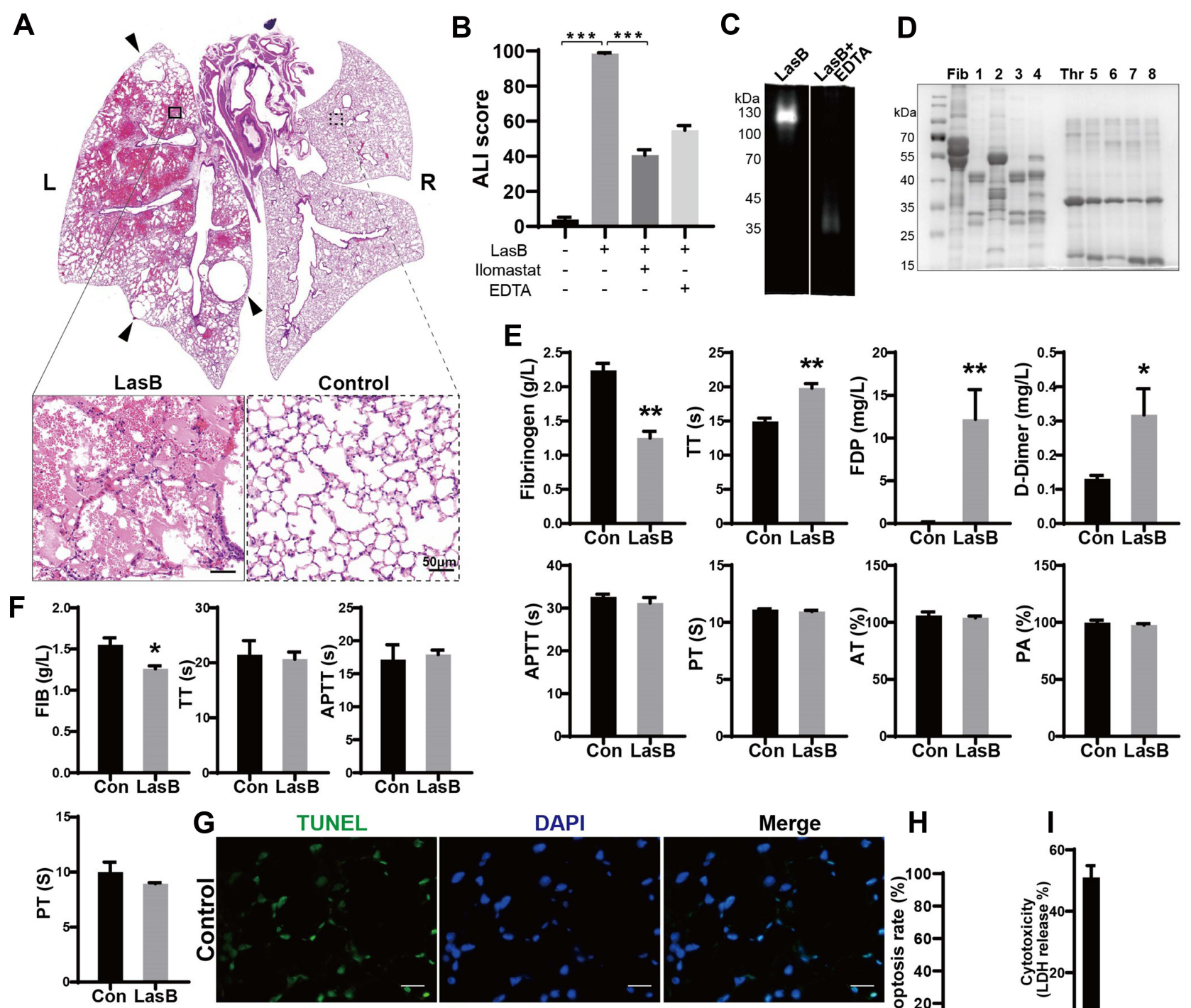

G

TUNEL

DAPI
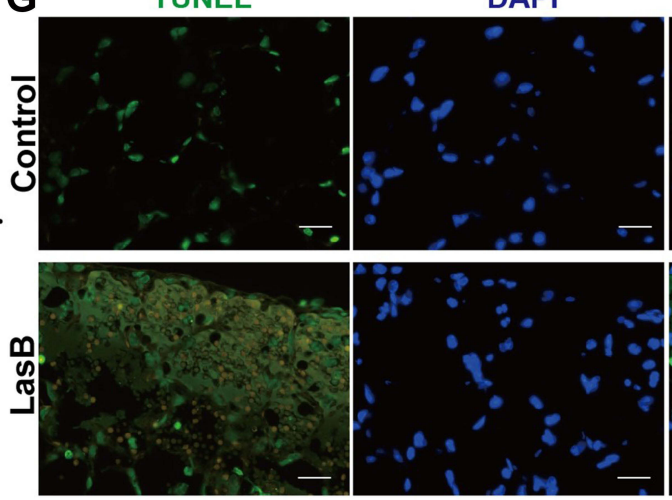
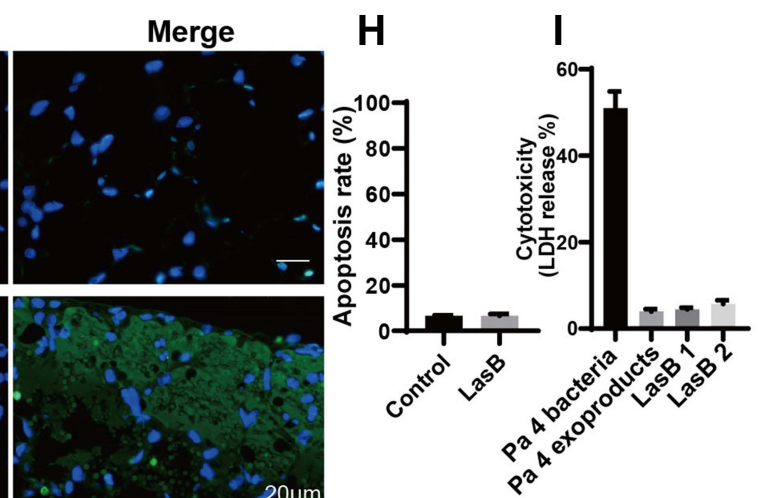

Figure 5 LasB elastase provokes hemorrhagic DAD, degrades extracellular matrix and causes hemostasis disorder (A) HE stain of mice lung unilaterally instilled with $3 \mu \mathrm{g}$ LasB elastase. DAD, hemorrhage, hyaline membranes, neutrophil infiltration and multiple bullae (arrowheads) occurred in LasB treated left lung; $L$, left; R, right. (B) ALI score of LasB injured lungs and the effect of different LasB inhibitors ( $n=3$ in each group). EDTA: 25 mM; llomastat: ImM. (C) Gelatin zymography of purified LasB and the inhibition effect of EDTA. (D) Degradation of fibrinogen and thrombin with or without inhibitors in vitro. Fib, fibrinogen; I: LasB + fibrinogen; 2 : LasB + ilomastat + fibrinogen; 3: LasB + TLCK + fibrinogen; 4: LasB + TLCK + fibrinogen; Thr, thrombin; 5: LasB + thrombin; 6: LasB + ilomastat + thrombin; 7: LasB + TLCK + thrombin; 8: LasB + TLCK + thrombin; (E) the effect of LasB on overall coagulation function. Blood from healthy donors was incubated with LasB for 35 min, room temperature. (F) The impact of LasB on coagulation function in vivo. Hemostasis of SD rats were measured I h after injection of LasB elastase through tail vein. (G) TUNEL stain of LasB injured lung tissue. No apparent cellular apoptosis was found. Cell nuclei (Blue), TUNEL-positive cells (Green). (H) Apoptosis rate of LasB injured lung cells. (I) Cytotoxicity assay of LasB, Pa 4 bacteria or exoproducts to THP-I cells. LasB I: $100 \mu \mathrm{g} / \mathrm{mL}$; LasB 2: $200 \mu \mathrm{g} / \mathrm{mL}$. *P $<0.05$; **P $<0.005 ; * * * \mathrm{P}<0.0005$.

factors, represented by T1SS, T2SS, T3SS and quorum sensing systems. Currently, T3SS, with its four toxins ExoU, ExoT, ExoS and ExoY, is recognized as the most important virulence factor of $P$. aeruginosa responsible for alveolar epithelial injury in patients. Strains possessing the exoU gene are especially hazardous. ${ }^{24}$ However, among the 8 strains tested, we found that the exoU+ genotype $\mathrm{Pa}$ 2 is not as virulent as the exoU-genotype $\mathrm{Pa} 4,5$ or 6 in causing DAD, indicating that LasB (T2SS) is probably more important than T3SS in ALI/ARDS pathogenesis.

Exoproducts of $P$. aeruginosa are composed of multiple pathogenic agents, such as alginate, LPS, flagellum, 
proteases from T2SS, exotoxins, pyocyanin, siderophores, hemolysins, and phospholipases, and each plays an important role in lung infection. ${ }^{25}$ Among them, we discovered that hemorrhagic DAD was mainly caused by proteases. However, $P$. aeruginosa secretes at least six proteases, including alkaline protease (AprA), elastase A (LasA), elastase B (LasB), large exoprotease A (LepA), protease IV (PIV) and Pseudomonas small protease (PASP). Many studies have reported that $P$. aeruginosa proteases cause severe hemorrhage or lung injury in animals, but the effect was never specifically attributed to LasB elastase, as opposed to LasA elastase or other proteases. ${ }^{26-30}$ Moreover, most studies investigated the role of LasB in lung infection using defined deletion or insertion mutants of the LasB gene, which may not be assigned unequivocally to the impact of LasB elastase. ${ }^{31-33}$ Instead, our team successfully purified $35 \mathrm{kDa}$ LasB elastase from a virulent $P$. aeruginosa strain and elucidated the direct causative effect of LasB on alveolar-capillary structure and clotting cascade in vivo and vitro. Furthermore, we ruled out the contribution of LasA, LepA, PASP and alkaline protease using a combined method of LC-MS/MS, SDS-PAGE and zymography. $^{13}$ We applied a TLCK inhibition assay to further exclude PIV contamination. Our study strongly suggests that LasB is the most important lung injury protease from $P$. aeruginosa.

$P$. aeruginosa LasB elastase (pseudolysin) is a neutral $\mathrm{Zn}^{2+}$-dependent metalloprotease with both proteolytic and elastolytic activities that can be inhibited by metal chelators, such as EDTA or MMP inhibitors. It has been shown to degrade a vast array of host proteins, including structural components, including elastin, collagen, laminin, immune factors such as IgA, IgG, TNF- $\alpha$, IFN- $\gamma$, IL-2, IL-6, and $\alpha 1$-antiprotease, and proteins involved in coagulation functions, such as fibrin, fibrinogen and thrombin. ${ }^{31}$ In this study, we validated that LasB is highly efficient in destroying structural proteins in the lung and key enzymes in clotting. Interestingly, although LasB per se is sufficient to provoke histopathological changes almost identical to DAD in ALI/ARDS, it did not show obvious cytotoxicity to pulmonary cells in vivo or to THP-1 cells in vitro. Jose et al reported similar results that moderate LasB protease is nontoxic to Hep2 cells. ${ }^{34}$ However, in real P. aeruginosa infection, not only DAD but also extended cell apoptosis were observed. Since bacteria are highly cytotoxic but not LasB and exoproducts, we suppose that cell death might be caused by T3SS or T6SSs, which are highly efficient but require direct bacteria-to-cell contact. ${ }^{24}$ Interestingly, we found that the most abundant protein secreted by less virulent $P$. aeruginosa strains was catalase, which probably explained their clinical prevalence because catalase offers strong protection over bacteria from $\mathrm{H}_{2} \mathrm{O}_{2}$ and antibioticmediated killing. ${ }^{35}$

FDPs and D-dimers are the products of degraded fibrinogen or fibrin. We discovered for the first time that LasB could significantly increase FDP and D-dimer levels and decrease fibrinogen levels in citrate-anticoagulated blood, causing DIC-like clotting dysfunction in vitro. Interestingly, LasB did not disrupt the anticoagulant process, which helped to explain the heavy bleeding in the lung. LasB administered intravenously to rats decreased fibrinogen levels in vivo as well. However, elongation of thrombin time was not found. We are not sure if this was caused by the strong compensatory capacity of the SD rats or by other reasons yet. More exploration will focus on this matter in the future.

In addition to $P$. aeruginosa, many bacteria secrete extracellular proteases, such as collagenase from Clostridium histolyticum or thermolysin from Bacillus thermoproteolyticus. $^{36}$ S. aureus, S. agalactiae and $S$. pneumoniae are also well known for their hyaluronidase production, ${ }^{37}$ which might have led to their unique pathological pattern in lung infection. Proteases enable bacteria with extraordinary power to break barriers and spread. ${ }^{36,38}$ However, due to limited time and resources, we were not able to test and identify the virulence factors of all clinically isolated bacteria. Additionally, as our research focused on the major respiratory pathogenic bacterial species in China, it is not clear whether our results are fully applicable to other areas. Thus, further studies are needed.

Zupetic et al recently proved that $P$. aeruginosa elastase activity was common in ICU respiratory isolates representing $75 \%$ of samples and was associated with increased 30 day mortality. ${ }^{39}$ In this study, we demonstrated that $P$. aeruginosa is the queen of pulmonary pathogens. Among all its exoproducts, LasB per se is sufficient and essential to elicit ARDS-like symptoms and histological changes in animals by inducing matrix degradation and coagulopathy. Monitoring LasB levels might be helpful in predicting the outcome of ALI/ARDS patients in the future.

\section{Acknowledgments}

We would like to thank Dr. Jingxian Liu (Division of Medical Microbiology, Department of Clinical Laboratory, Xinhua Hospital) as well as Professor Yong Zhang (Department of Immunology, SJTUSM) and Professor Min 
Wang (Department of Histology and Embryology, SJTUSM) for their skilled advice and assistance.

\section{Author Contributions}

All authors contributed to data analysis, drafting or revising the article, gave final approval of the version to be published, agreed to the submitted journal, and agree to be accountable for all aspects of the work.

\section{Funding}

National Natural Science Foundation of China. NSFC NO.81772111.

\section{Disclosure}

The authors declare no conflicts of interest in this work.

\section{References}

1. Fan E, Brodie D, Slutsky AS. Acute respiratory distress syndrome: advances in diagnosis and treatment. JAMA. 2018;319(7):698-710. doi:10.1001/jama.2017.21907

2. Silva PL, Pelosi P, Rocco PRM. Personalized pharmacological therapy for ARDS: a light at the end of the tunnel. Expert Opin Investig Drugs. 2020;29(1):49-61. doi:10.1080/13543784.2020.1699531

3. Bellani G, Laffey JG, Pham T, et al. Epidemiology, patterns of care, and mortality for patients with acute respiratory distress syndrome in intensive care units in 50 countries. JAMA. 2016;315(8):788-800. doi:10.1001/jama.2016.0291

4. Tanner JR, Kingsley RA. Evolution of salmonella within hosts. Trends Microbiol. 2018;26(12):986-998. doi:10.1016/j.tim.2018.06.001

5. Defraine V, Fauvart M, Michiels J. Fighting bacterial persistence: current and emerging anti-persister strategies and therapeutics. Drug Resist Updat. 2018;38:12-26. doi:10.1016/j.drup.2018.03.002

6. Bauer TT, Ewig S, Rodloff AC, et al. Acute respiratory distress syndrome and pneumonia: a comprehensive review of clinical data. Clin Infect Dis. 2006;43(6):748-756. doi:10.1086/506430

7. Cepas V, Soto SM. Relationship between virulence and resistance among gram-negative bacteria. Antibiotics. 2020;9(10). doi:10.3390/ antibiotics 9100719

8. Maunders EA, Triniman RC, Western J, et al. Global reprogramming of virulence and antibiotic resistance in Pseudomonas aeruginosa by a single nucleotide polymorphism in elongation factor, fusA1. $J$ Biol Chem. 2020;295(48):16411-16426. doi:10.1074/jbc.RA119.012102

9. Duan Y, Gao H, Zheng L, et al. Antibiotic resistance and virulence of Extraintestinal Pathogenic Escherichia coli (ExPEC) vary according to molecular types. Front Microbiol. 2020;11:598305. doi:10.3389/ fmicb.2020.598305

10. Hu F, Guo Y, Yang Y, et al. Resistance reported from China antimicrobial surveillance network (CHINET) in 2018. Eur J Clin Microbiol Infect Dis. 2019;38(12):2275-2281. doi:10.1007/s10096019-03673-1

11. Matute-Bello G, Downey G, Moore BB, et al. An official American thoracic society workshop report: features and measurements of experimental acute lung injury in animals. Am J Respir Cell Mol Biol. 2011;44(5):725-738. doi:10.1165/rcmb.2009-0210ST

12. Hsia CCW, Hyde DM, Ochs M, et al. An official research policy statement of the American thoracic society/European respiratory society: standards for quantitative assessment of lung structure. $\mathrm{Am}$ J Respir Crit Care Med. 2010;181(4):394-418. doi:10.1164/ rccm.200809-1522ST
13. Kessler E, Safrin M. Elastinolytic and proteolytic enzymes. Methods Mol Biol. 2014;1149:135-169. doi:10.1007/978-1-4939-0473-0_13

14. Wiśniewski JR, Zougman A, Nagaraj N, et al. Universal sample preparation method for proteome analysis. Nat Methods. 2009;6 (5):359-362. doi:10.1038/nmeth.1322

15. Rubenfeld GD, Thompson T, Ferguson ND, et al. Acute respiratory distress syndrome: the berlin definition. JAMA. 2012;307 (23):2526-2533. doi:10.1001/jama.2012.5669

16. Deshpande R, Zou C. Pseudomonas aeruginosa induced cell death in acute lung injury and acute respiratory distress syndrome. Int $J \mathrm{Mol}$ Sci. 2020;21(15):5356. doi:10.3390/ijms21155356

17. Marquart ME, Caballero AR, Chomnawang M, et al. Identification of a novel secreted protease from Pseudomonas aeruginosa that causes corneal erosions. Invest Ophthalmol Vis Sci. 2005;46(10):3761-3768. doi:10.1167/iovs.04-1483

18. Caballero AR, Moreau JM, Engel LS, et al. Pseudomonas aeruginosa protease IV enzyme assays and comparison to other Pseudomonas proteases. Anal Biochem. 2001;290(2):330-337. doi:10.1006/ abio.2001.4999

19. Rawson TM, Moore LS, Zhu N, et al. Bacterial and fungal coinfection in individuals with coronavirus: a rapid review to support COVID-19 antimicrobial prescribing. Clin Infect Dis. 2020;71 (9):2459-2468. doi:10.1093/cid/ciaa530

20. Bakaletz LO. Viral-bacterial co-infections in the respiratory tract. Curr Opin Microbiol. 2017;35:30-35. doi:10.1016/j.mib.2016.11.003

21. Mannes GP, Boersma WG, Baur CH, Postmus PE. Adult respiratory distress syndrome (ARDS) due to bacteraemic pneumococcal pneumonia. Eur Respir J. 1991;4(4):503-504.

22. Trautmann M, Lepper PM, Haller M. Ecology of Pseudomonas aeruginosa in the intensive care unit and the evolving role of water outlets as a reservoir of the organism. Am J Infect Control. 2005;33 (Suppl 5):S41-S49. doi:10.1016/j.ajic.2005.03.006

23. Botelho J, Grosso F, Peixe L. Antibiotic resistance in Pseudomonas aeruginosa - mechanisms, epidemiology and evolution. Drug Resist Updat. 2019;44:100640. doi:10.1016/j.drup.2019.07.002

24. Sawa T, Shimizu M, Moriyama K, et al. Association between Pseudomonas aeruginosa type III secretion, antibiotic resistance, and clinical outcome: a review. Crit Care. 2014;18(6):668. doi:10.1186/s13054-014-0668-9

25. Sauvage S, Hardouin J. Exoproteomics for Better understanding Pseudomonas aeruginosa virulence. Toxins. 2020;12(9):571. doi:10.3390/toxins 12090571

26. Meinke G, Barum J, Rosenberg B, et al. In vivo studies with the partially purified protease (elastase) from Pseudomonas aeruginosa. Infect Immun. 1970;2(5):583-589. doi:10.1128/iai.2.5.583-589.1970

27. Elsen S, Huber P, Bouillot S, et al. A type III secretion negative clinical strain of Pseudomonas aeruginosa employs a two-partner secreted exolysin to induce hemorrhagic pneumonia. Cell Host Microbe. 2014;15(2):164-176. doi:10.1016/j.chom.2014.01.003

28. Williams JC, Lucas BJ, Knee C, Renzetti M, Donahue J. Acute lung injury induced by Pseudomonas aeruginosa elastase in hamsters. Exp Lung Res. 1992;18(1):155-171. doi:10.3109/01902149209020658

29. Gray L, Kreger A. Microscopic characterization of rabbit lung damage produced by Pseudomonas aeruginosa proteases. Infect Immun. 1979;23(1):150-159. doi:10.1128/iai.23.1.150-159.1979

30. Wolz C, Hellstern E, Haug M, et al. Pseudomonas aeruginosa LasB mutant constructed by insertional mutagenesis reveais elastolytic activity due to alkaline proteinase and the LasA fragment. Mol Microbiol. 1991;5(9):2125-2131. doi:10.1111/j.1365-2958.1991. tb02142.x

31. Everett MJ, Davies DT. Pseudomonas aeruginosa elastase (LasB) as a therapeutic target. Drug Discov Today. 2021. doi:10.1016/j. drudis.2021.02.026

32. Qu Y, Olonisakin T, Bain W, et al. Thrombospondin-1 protects against pathogen-induced lung injury by limiting extracellular matrix proteolysis. JCI Insight. 2018;3(3). doi:10.1172/jci.insight.96914 
33. Cowell BA, Twining SS, Hobden JA, et al. Mutation of lasA and lasB reduces Pseudomonas aeruginosa invasion of epithelial cells. Microbiology. 2003;149(8):2291-2299. doi:10.1099/mic.0.26280-0

34. Jose D, Manjusha K, Jose S, Mohandas A, Singh IS. Purification and characterization of highly active LasB protease from pseudomonas aeruginosa MCCB 123. Indian J Exp Biol. 2017;55:303-310.

35. Khakimova M, Ahlgren HG, Harrison JJ, et al. The stringent response controls catalases in Pseudomonas aeruginosa and is required for hydrogen peroxide and antibiotic tolerance. $J$ Bacteriol. 2013;195(9):2011-2020. doi:10.1128/jb.02061-12

36. Duarte AS, Correia A, Esteves AC. Bacterial collagenases - a review. Crit Rev Microbiol. 2016;42(1):106-126. doi:10.3109/1040841x.20 14.904270
37. Hynes WL, Walton SL. Hyaluronidases of gram-positive bacteria. FEMS Microbiol Lett. 2000;183(2):201-207. doi:10.1111/j.15746968.2000.tb08958.x

38. Matsumoto K. Role of bacterial proteases in pseudomonal and serratial keratitis. Biol Chem. 2004;385(11):1007-1016. doi:10.1515/ bc. 2004.131

39. Zupetic J, Peñaloza HF, Bain W, et al. Elastase activity from Pseudomonas aeruginosa respiratory isolates and ICU mortality. Chest. 2021. doi:10.1016/j.chest.2021.04.015

\section{Publish your work in this journal}

The Journal of Inflammation Research is an international, peerreviewed open-access journal that welcomes laboratory and clinical findings on the molecular basis, cell biology and pharmacology of inflammation including original research, reviews, symposium reports, hypothesis formation and commentaries on: acute/chronic inflammation; mediators of inflammation; cellular processes; molecular mechanisms; pharmacology and novel anti-inflammatory drugs; clinical conditions involving inflammation. The manuscript management system is completely online and includes a very quick and fair peerreview system. Visit http://www.dovepress.com/testimonials.php to read real quotes from published authors. 